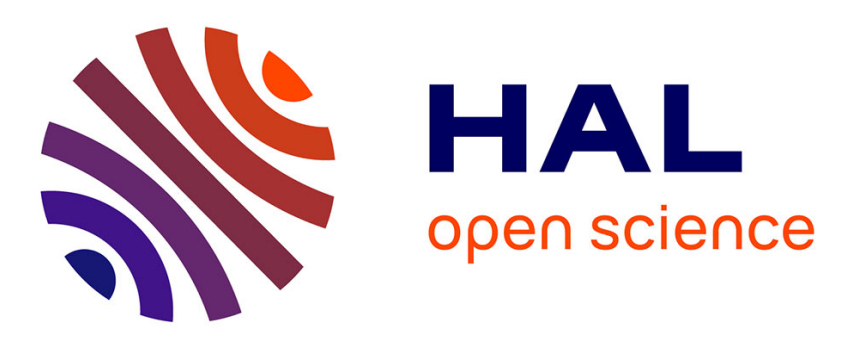

\title{
Contraction and Tumbling Dynamics of DNA in Shear Flows under Confinement Induced by Transverse Viscoelastic Forces
}

Marius Socol, Hubert Ranchon, Bayan Chami, Antony Lesage, Jean-Marc Victor, Manoel Manghi, Aurélien Bancaud

\section{To cite this version:}

Marius Socol, Hubert Ranchon, Bayan Chami, Antony Lesage, Jean-Marc Victor, et al.. Contraction and Tumbling Dynamics of DNA in Shear Flows under Confinement Induced by Transverse Viscoelastic Forces. Macromolecules, 2019, 52 (4), pp.1843-1852. 10.1021/acs.macromol.8b02184 . hal-02076283

\section{HAL Id: hal-02076283 https://hal.science/hal-02076283}

Submitted on 4 Jul 2019

HAL is a multi-disciplinary open access archive for the deposit and dissemination of scientific research documents, whether they are published or not. The documents may come from teaching and research institutions in France or abroad, or from public or private research centers.
L'archive ouverte pluridisciplinaire HAL, est destinée au dépôt et à la diffusion de documents scientifiques de niveau recherche, publiés ou non, émanant des établissements d'enseignement et de recherche français ou étrangers, des laboratoires publics ou privés. 


\title{
Contraction and tumbling dynamics of DNA
} in shear flows under confinement induced by transverse viscoelastic forces

\author{
Marius Socol, ${ }^{\dagger}$ Hubert Ranchon, ${ }^{\dagger}$ Bayan Chami, $^{\dagger}$ Antony Lesage,$^{\ddagger}$ Jean-Marc \\ Victor, $\ddagger$ Manoel Manghi, ${ }^{*} \uparrow$ and Aurélien Bancaud ${ }^{* \dagger}$ \\ $\dagger L A A S-C N R S$, Université de Toulouse, CNRS, F-31400 Toulouse, France \\ †Sorbonne Université, CNRS, Laboratoire de Physique Théorique de la Matière Condensée, \\ LPTMC, F-75005 Paris, France \\ \Laboratoire de Physique Théorique (IRSAMC), Université de Toulouse, CNRS, UPS, \\ Toulouse, France \\ E-mail: manghi@irsamc.ups-tlse.fr; abancaud@laas.fr
}




\begin{abstract}
The dynamics of single DNA molecules conveyed in a viscoelastic fluid flow with an opposing electrophoretic force are investigated by fluorescence microscopy. For balanced hydrodynamic and electrophoretic forces, DNA is confined near the walls with a much smaller elongation than in bulk shear flows. Furthermore, we observe that DNA extension is characterized by intermittent fluctuations, the characteristic time scale of which depends on the flow velocity. A model based on Rouse dynamics explains the contraction of the molecule by the coupling of monomer motion in the transverse and longitudinal directions to the flow induced by transverse viscoelastic forces. Using simulations, we suggest that the occurrence of intermittent fluctuations is analogous to tumbling dynamics characterized by the cyclic spooling motion of end monomers about the molecule center of mass.
\end{abstract}




\section{Introduction}

The conformational response of a polymer molecule is highly dependent on the type of flow. ${ }^{1,2}$ In a pure elongational flow, macromolecules are highly extended, ${ }^{3}$ whereas in a simple shear flow where elongation and rotation coexist, the rotational component forces polymers to tumble. ${ }^{4}$ As the shear rate $\dot{\gamma}$ of the flow increases, the average extension of the polymer molecule increases, ${ }^{5}$ but tumbling prevents it from reaching a steady elongated conformation. The periodic tumbling motion is dictated by the shear rate of the flow, and can be recapitulated by advection and diffusion arguments. ${ }^{6}$ In pressure-driven flows with a Poiseuille profile, the shear rate varies across the channel cross-section. In this case, in addition to tumbling dynamics, which have been analyzed using arguments similar to those of shear flows, ${ }^{7,8}$ transverse migration of polymers and polyelectrolytes toward the centreline of the channel has been detected. ${ }^{9-12}$

Confinement fundamentally alters the folding and transport properties of dilute polymer solutions. ${ }^{13}$ For instance, steric repulsion forbids the occurrence of end-over-end tumbling if the contour length of the molecule is greater than the channel height. Experiments and simulations have shown that DNA molecules are stretched along the channel and squeezed in the transverse direction for high levels of confinement. ${ }^{14,15}$ Although simulations have hinted to the existence of strong differences in deformation and tumbling time distribution between Poiseuille and simple shear flows for strongly confined polymers, ${ }^{14}$ the physics of DNA in confined shear flows has not been studied experimentally. We recently showed that DNA molecules conveyed in viscoelastic fluid flows with an opposing electrophoretic force were dragged by a viscoelastic force towards the walls of micro-channels. ${ }^{16}$ Hence, transverse migration sets the molecules under confinement in a shear flow. In this report, we analyze the configuration and dynamics of fluorescently labelled DNA at the single molecule level in this regime. In contrast to the large elongation observed in bulk shear flows, we report that DNA adopts a contracted conformation, which is studied both analytically and using simulations. 


\section{Materials and Methods}

\section{Sample preparation}

Molecular biology grades chemicals were purchased from Sigma-Aldrich (St. Louis, MO), bacteriophage- $\lambda$ DNA (48,504 bp) from New England Biolabs (Ipswich, MA), and the fluorophore YOYO-1 from ThermoFisher (Waltham, MA). Our experiments were carried out with $\lambda$-DNA molecules stained with YOYO-1 with a labelling ratio of 5 base pairs per dye molecule. YOYO-1 intercalation overstretches DNA by a factor of $28 \%$ with minor change in persistence length, ${ }^{17}$ leading to a contour length of $21.1 \mu \mathrm{m}$. Macromolecules were diluted at a concentration of $0.1 \mathrm{ng} / \mu \mathrm{L}$ in a viscoelastic buffer composed of $2 \%(\mathrm{~m} / \mathrm{v})$ polyvinylpyrrolidone (PVP $360 \mathrm{kDa}$ ) dissolved in 1x TBE (Tris-HCl 89 mM, Boric acid $89 \mathrm{mM}$, EDTA $2 \mathrm{mM})$ supplemented with $5 \%(\mathrm{~m} / \mathrm{v})$ dithiothreitol to reduce photodegradation. Note that $2 \%$ PVP corresponds to three times the overlapping concentration of this polymer. ${ }^{18}$ The viscosity $\eta$ and relaxation time $\tau$ of the solution were $5.5 \mathrm{mPa} . \mathrm{s}$ and $20 \mathrm{~ms}$, respectively, according to our calibration method described in Ref. ${ }^{19}$

\section{Microfluidic chip fabrication}

Microfluidic channels were fabricated in 4-inch silicon wafers that were processed with a first step of reactive ion etching down to a depth of 0.35 to $2 \mu \mathrm{m}$, followed by access holes drilling by sand blasting, dry oxidation over a thickness of $200 \mathrm{~nm}$, and anodic bonding with $160 \mu \mathrm{m}$ glass wafers, as described in Ref. ${ }^{20}$ Note that the shallower channels were etched an additional time to engrave thicker channels of $20 \mu \mathrm{m}$ near access holes in order to reduce the hydrodynamic resistance of the chip. The resulting microfluidic chips, sketched in Fig. 1A, had a slit geometry characterized by a width (in the $y$ direction) of $200 \mu \mathrm{m}$, a narrow height, noted $h$, (in the $z$ direction), and a length of $2 \mathrm{~cm}$ (in the $x$ direction). 


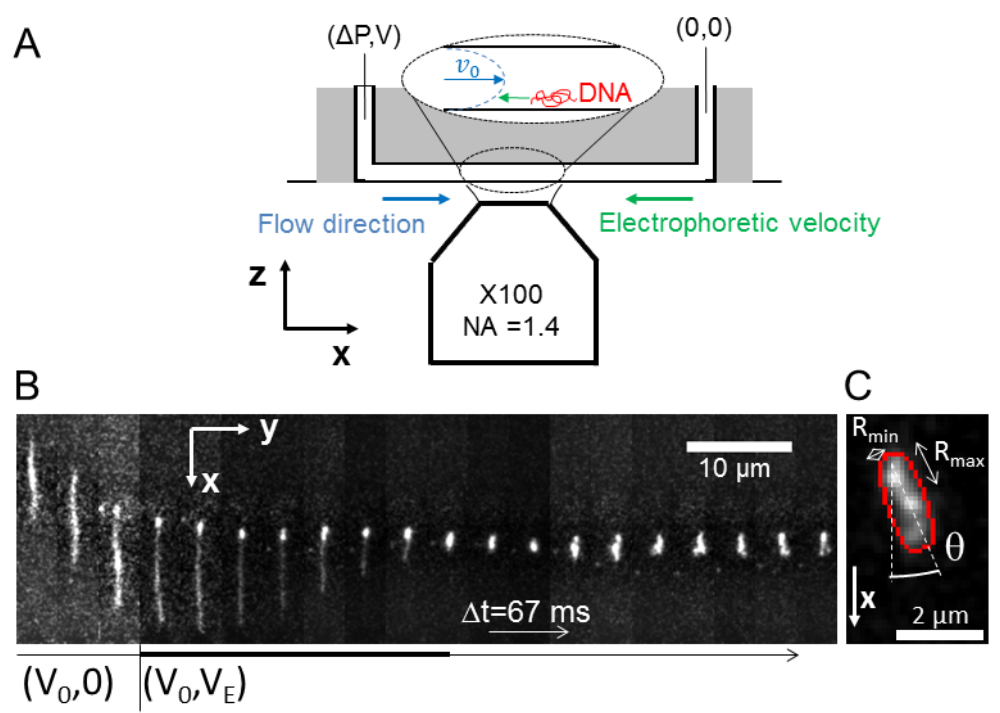

Figure 1: Experiment design: (A) Overview of the experiment with a slit-like microfluidic channel in which $\lambda$-DNA molecules are conveyed by a pressure-driven hydrodynamic flow and a counter electrophoretic force, shown with blue and green arrows, respectively. $\Delta P$ and $V$ are the pressure and potential drops between the left and the right reservoirs. (B) The time lapse shows the response of a DNA molecule first in a simple hydrodynamic flow (three first images) and then with electro-hydrodynamic actuation. The first eight images underlined with a thick line along the time axis correspond to the transient relaxation phase. The flow and electrophoretic velocities are $V_{0}=290 \mu \mathrm{m} / \mathrm{s}$, as inferred from particle velocimetry using the NVDA method, ${ }^{20}$ and $V_{E}=43 \mu \mathrm{m} / \mathrm{s}$, respectively. The electrophoretic velocity $V_{E}$ is measured by tracking single $\lambda$-DNA molecules in absence of flow (not shown). (C) Conformation analysis of single molecule based on gray-value weighted gyration matrix analysis. $^{21}$

\section{Apparatus and image processing}

DNA molecules were conveyed by a pressure-driven flow (in the $x$ direction) using a controller operating in the 0.1 to 3 bar range (Fluigent, France), as well as by electrophoresis in the opposite direction with a DC supply generating 10-250 V (Keysight, CA). Time lapse fluorescence micrographs were recorded using a Zeiss epifluorescence wide field microscope equipped with a 100X objective with a numerical aperture of 1.4 and a Zyla sCMOS camera (Andor, Ireland). ${ }^{16}$ A typical time series is reported in Fig. 1B. The molecules were segmented in ImageJ using the "Maximum Entropy" threshold. We then extracted the center of mass in order to infer the molecule velocity, as well as its conformation based on the gray-value 
weighted gyration matrix to measure the major axis $R_{\max }$, the minor axis $R_{\min }$, and the tilt angle $\theta$ with respect to the flow direction (Fig. 1C). ${ }^{21}$ The shear rate was made dimensionless by expressing $\dot{\gamma}$ as the Weissenberg number $W i=\dot{\gamma} \tau_{0}$, where $\tau_{0}$ is the longest polymer relaxation time. We measured $\tau_{0}$ by timing the rotational relaxation of identical molecules at rest in the channels and averaging. ${ }^{22}$ The standard deviation of these measurements is $\simeq 10 \%$.

\section{Brownian Dynamics Simulations}

We performed Brownian dynamics simulations of a system of 164 interconnected particles that were subjected to inter-particle elastic forces, transverse viscoelastic forces, and fluctuations. We neglected hydrodynamic interactions and inter-particle repulsion. This model is therefore used in a Rouse bead-spring description of flexible polymer molecules (see model section). The position of each Brownian particle, in interaction with its nearest neighbors, is governed by the Langevin equation. The simulations were run in Matlab using a code similar to that described in Ref. ${ }^{20}$ Simulations started with a linear configuration along the $x$ axis. The time step of the simulation was $1 \mathrm{~ms}$. The first phase of the simulation was a relaxation to the equilibrium conformation near the wall, which took place in 25,000 time steps. We started recording the dynamics of the molecule after 50,000 time steps during 450,000 time steps.

\section{Experimental observation of contraction}

First, we report the response of a molecule initially conveyed by hydrodynamics in a channel of height $h=2 \mu \mathrm{m}$ (three first images in Fig. 1B). Its convection (in the $x$ direction of the flow) can be detected from the drift of its center of mass, and its conformation is expectedly extended in the confined Poiseuille flow, ${ }^{14} \mathbf{v}(z)=4 V_{0} z / h(1-z / h) \mathbf{e}_{x}$ where $V_{0}$ is the flow velocity at the channel center imposed by the pressure drop. From our measure of the DNA 
relaxation time $\tau_{0}=0.17 \mathrm{~s}$ (for $V_{0}=V_{E}=0$, in agreement with Ref., ${ }^{22}$ see calibration below), and the shear rate close to the wall $\dot{\gamma}=4 V_{0} / h$, the Weissenberg number is, in this case for $V_{0}=290 \mu \mathrm{m} / \mathrm{s}, W i \simeq 80$. As the electric field is turned on starting from the fourth image in Fig. 1B, the molecule migration is stalled, and after a transient period of $\simeq 0.25 \mathrm{~s}$, it appears to contract in the field of view of the microscope with a typical end-to-end distance of $\sim 2 \mu \mathrm{m}$ (Fig. 1B-C). This contracted state, which is detected for every channel height $h$, appears to be relatively stable over time with some fluctuations mostly along the flow direction.

\section{Model}

\section{Rouse model}

In the absence of electrophoresis, the existence of transverse viscoelastic forces oriented toward the channel centerline have been extensively described for particles and for DNA molecules. ${ }^{23,24}$ Assuming a Maxwellian fluid (with a relaxation time $\tau$ ), the transverse viscoelastic force $F_{V E}$ exerted on a sphere of radius a reads:

$$
\mathbf{F}_{V E}(z)=-2 \pi K \eta \tau a^{3} \frac{\partial \dot{\gamma}^{2}}{\partial z} \mathbf{e}_{z}
$$

where $K$ is a constant on the order of $10^{-1}, z$ the distance from the wall, and $\dot{\gamma}$ the flow shear rate. However, in the case where an electrophoretic velocity $\mathbf{v}_{E}=-V_{E} \mathbf{e}_{x}$ is applied to the DNA, this force is oriented towards the walls of the channel ( $z=0$ or $z=h$ ). By assuming a linear drop of the force close to the nearest wall (we choose here $z=0$ ), we obtained the following expression: ${ }^{19}$

$$
F_{V E}(z) \simeq-32 \pi K^{\prime} \eta \tau \frac{L}{h} V_{0} V_{E} \frac{z}{h} \equiv-\Gamma z
$$

where $K^{\prime}$ is a constant on the order of $10^{-2}$, and $L$ the contour length of the molecule. This expression, which is analogous to that exerted by a spring to maintain the molecule near the 
wall, was verified experimentally. ${ }^{19}$

To gain further insight into the physics of contraction, we study the chain dynamics by modelling the DNA as a polymer following the Rouse dynamics in a shear flow, submitted to the transverse viscoelastic force given in Eq. (2), $\mathbf{F}_{V E}=-\Gamma z \mathbf{e}_{z}$, and the electrophoretic velocity $\mathbf{v}_{E}$. The position of the $n^{\text {th }}$ monomer, $\mathbf{r}_{n}$, is governed by the overdamped Langevin equation $(n=1, \ldots, N):^{25}$

$$
\xi \frac{\mathrm{d} \mathbf{r}_{n}}{\mathrm{~d} t}=-k\left(2 \mathbf{r}_{n}-\mathbf{r}_{n-1}-\mathbf{r}_{n+1}\right)-\xi V_{E} \mathbf{e}_{x}+\xi \dot{\gamma} z_{n} \mathbf{e}_{x}-\Gamma z_{n} \mathbf{e}_{z}+\mathbf{f}_{n}
$$

with $\mathbf{r}_{0}=\mathbf{r}_{1}$ and $\mathbf{r}_{N+1}=\mathbf{r}_{N}$. The consecutive terms in the right hand side are the sum of elastic nearest-neighbor forces with $k=3 k_{\mathrm{B}} T / b^{2}$ the entropic spring constant $\left(k_{\mathrm{B}} T\right.$ is the thermal energy and $b$ the polymer Kuhn length), the electrophoretic force, the shear force determined by the shear rate $\dot{\gamma}=4 V_{0} / h$, the viscoelastic force and random forces. Random forces are characterized by $\left\langle\mathbf{f}_{n}(t)\right\rangle=0$ and $\left.\left\langle\mathbf{f}_{n}(t) \cdot \mathbf{f}_{m}\left(t^{\prime}\right)\right\rangle\right\rangle=6 \xi k_{\mathrm{B}} T \delta_{n m} \delta\left(t-t^{\prime}\right)$ where $\xi=3 \pi \eta b$ is the monomer friction coefficient. We neglected the bending energy for simplicity of the calculations, and assumed that intramolecular hydrodynamic interactions are negligible due to the confinement of the molecule near the walls. Moreover we do not model the hard-core and electrostatic repulsion between the polymer and the substrate at $z=0$. We therefore assume that the confined equilibrium position $\langle z\rangle$ is larger than the range of these interactions. This Langevin equation is also used to perform Brownian dynamics simulations.

By replacing the first term of the right hand side by $k \partial^{2} \mathbf{r}_{n} / \partial n^{2}$ in the continuous limit, and using the Fourier decomposition, $\hat{\mathbf{r}}_{p}(t)=\frac{1}{N} \int_{0}^{N} d n \mathbf{r}_{n}(t) \cos \left(\frac{p n \pi}{N}\right)$, Eq. (3) becomes

$$
\begin{aligned}
\frac{\partial \hat{x}_{p}}{\partial t} & =-\frac{k_{p}}{\xi_{p}} \hat{x}_{p}-V_{E} \delta_{p 0}+\dot{\gamma} \hat{z}_{p}+\frac{\hat{f}_{p x}}{\xi_{p}} \\
\frac{\partial \hat{y}_{p}}{\partial t} & =-\frac{k_{p}}{\xi_{p}} \hat{y}_{p}+\frac{\hat{f}_{p y}}{\xi_{p}}
\end{aligned}
$$




$$
\frac{\partial \hat{z}_{p}}{\partial t}=-\frac{k_{p}}{\xi_{p}} \hat{z}_{p}-\frac{\Gamma}{\xi} \hat{z}_{p}+\frac{\hat{f}_{p z}}{\xi_{p}}
$$

where $k_{p}=2 \pi^{2} \frac{k}{N} p^{2}$ and $\xi_{p}=2 N \xi$ for $p=1,2 \ldots\left(\xi_{0}=N \xi\right)$, and $\left\langle\hat{f}_{p i}(t) \hat{f}_{q j}(t)\right\rangle=2 \xi_{p} k_{\mathrm{B}} T \delta(t-$ $\left.t^{\prime}\right) \delta_{p q} \delta_{i j}$. We note the Rouse times $\tau_{p}=\xi_{p} / k_{p}=\tau_{1} / p^{2}$ with $\tau_{1}=\xi N^{2} /\left(\pi^{2} k\right)=\eta L^{2} b /\left(\pi k_{\mathrm{B}} T\right)$ the longest one. ${ }^{25}$ The other characteristic time is $\tau_{p}^{\prime}$ defined through

$$
\frac{1}{\tau_{p}^{\prime}}=\frac{1}{\tau_{p}}+\frac{\Gamma}{\xi}=\frac{1}{\tau_{p}}\left(1+\frac{a}{p^{2}}\right) \quad \text { where } \quad a=\frac{\tau_{1} \Gamma}{\xi}
$$

The parameter $a=\tau_{1} / \tau_{\Gamma}=N^{2} \Gamma /\left(\pi^{2} k\right)$ is simply the ratio of the (longest) Rouse time with the relaxation time $\tau_{\Gamma}=\xi / \Gamma$ in the harmonic potential induced by viscoelastic forces. If we assume a Kuhn length of $b=2 \ell_{\mathrm{p}}$ where $\ell_{\mathrm{p}} \simeq 50 \mathrm{~nm}$ at the experimental salt concentration $^{26}$ and take into account the stretching of the molecule by YOYO-1 fluorophore to $L=21.1 \mu \mathrm{m},{ }^{17}$ we obtain $N=211$ and a Rouse time $\tau_{1} \simeq 19.5 \mathrm{~s}$.

The auto-correlation functions for the $y$ and $z$ components are therefore

$$
\begin{aligned}
\left\langle\hat{y}_{p}(t) \hat{y}_{p}(0)\right\rangle & =\frac{k_{\mathrm{B}} T}{k_{p}} e^{-t / \tau_{p}} \\
\left\langle\hat{z}_{p}(t) \hat{z}_{p}(0)\right\rangle & =\frac{k_{\mathrm{B}} T}{k_{p}\left(1+a / p^{2}\right)} e^{-t / \tau_{p}^{\prime}}
\end{aligned}
$$

To compute the last one $\left\langle\hat{x}_{p}(t) \hat{x}_{p}(0)\right\rangle$, we can show that the coordinate $\hat{X}_{p} \equiv \hat{x}_{p}+\frac{\dot{\gamma} \tau_{1}}{a} \hat{z}_{p}$ obeys the following equation

$$
\frac{\partial \hat{X}_{p}}{\partial t}=-\frac{k_{p}}{\xi_{p}} \hat{X}_{p}+\frac{\hat{f}_{p X}}{\xi_{p}}
$$

with $\left\langle\hat{f}_{p X}(t) \hat{f}_{q X}(t)\right\rangle=2 \xi_{p}\left[1+\left(\frac{\dot{\gamma} \tau_{1}}{a}\right)^{2}\right] k_{\mathrm{B}} T \delta\left(t-t^{\prime}\right) \delta_{p q}$. Hence we find

$$
\begin{aligned}
\left\langle\hat{X}_{p}(t) \hat{X}_{p}(0)\right\rangle & =\frac{k_{\mathrm{B}} T}{k_{p}}\left[1+\left(\frac{\dot{\gamma} \tau_{1}}{a}\right)^{2}\right] e^{-t / \tau_{p}} \\
& =\left\langle\hat{x}_{p}(t) \hat{x}_{p}(0)\right\rangle+2 \frac{\dot{\gamma} \tau_{1}}{a}\left\langle\hat{z}_{p}(t) \hat{x}_{p}(0)\right\rangle+\left(\frac{\dot{\gamma} \tau_{1}}{a}\right)^{2}\left\langle\hat{z}_{p}(t) \hat{z}_{p}(0)\right\rangle
\end{aligned}
$$


The last step consists in computing the cross-correlation function $\left\langle\hat{z}_{p}(t) \hat{x}_{p}(0)\right\rangle=\frac{\dot{\gamma} \tau_{1}}{2 p^{2}+a}\left\langle\hat{z}_{p}(t) \hat{z}_{p}(0)\right\rangle$. Gathering the results, we finally find

$$
\left\langle\hat{x}_{p}(t) \hat{x}_{p}(0)\right\rangle=\frac{k_{\mathrm{B}} T}{k_{p}}\left\{\left[1+\left(\frac{\dot{\gamma} \tau_{1}}{a}\right)^{2}\right] e^{-t / \tau_{p}}-\left(\frac{\dot{\gamma} \tau_{1}}{a}\right)^{2} \frac{p^{2}\left(3 a+2 p^{2}\right)}{\left(a+p^{2}\right)\left(a+2 p^{2}\right)} e^{-t / \tau_{p}^{\prime}}\right\}
$$

To describe the steady-state conformation of the polymer, it is usual to compute the shape tensor, ${ }^{27}$ defined as

$$
I_{i j}=\frac{1}{N} \sum_{n=1}^{N}\left\langle\left(\mathbf{r}_{n}-\mathbf{R}_{G}\right)_{i}\left(\mathbf{r}_{n}-\mathbf{R}_{G}\right)_{j}\right\rangle
$$

where $\mathbf{R}_{G}=\frac{1}{N} \sum_{n=1}^{N} \mathbf{r}_{n}$ is the position of the center of mass of the chain. Noting that $\mathbf{R}_{G}=$ $\hat{\mathbf{r}}_{0}$, we can easily rewrite $I_{i j}=2 \sum_{p=1}^{\infty}\left\langle\hat{\mathbf{r}}_{p i}(0) \hat{\mathbf{r}}_{p j}(0)\right\rangle$. The four non-vanishing components are

$$
\begin{aligned}
I_{y y} & =2 \sum_{p=1}^{\infty} \frac{k_{\mathrm{B}} T}{k_{p}}=\frac{N b^{2}}{18} \\
I_{z z} & =\frac{N b^{2}}{6 \pi \sqrt{a}}\left[\operatorname{coth}(\pi \sqrt{a})-\frac{1}{\pi \sqrt{a}}\right] \\
I_{x z} & =\dot{\gamma} \tau_{1} \frac{N b^{2}}{3 \pi^{2}} \sum_{p=1}^{\infty} \frac{1}{\left(a+p^{2}\right)\left(a+2 p^{2}\right)} \\
& =\frac{\dot{\gamma} \tau_{1}}{a^{3 / 2}} \frac{N b^{2}}{6 \pi}\left[\sqrt{2} \operatorname{coth}\left(\pi \sqrt{\frac{a}{2}}\right)-\operatorname{coth}(\pi \sqrt{a})-\frac{1}{\pi \sqrt{a}}\right] \\
I_{x x} & =\frac{N b^{2}}{18}\left[1+\left(\dot{\gamma} \tau_{1}\right)^{2} \frac{6}{\pi^{2}} \sum_{p=1}^{\infty} \frac{1}{p^{2}\left(a+p^{2}\right)\left(a+2 p^{2}\right)}\right] \\
& =\frac{N b^{2}}{18}\left\{1+\left(\frac{\dot{\gamma} \tau_{1}}{a}\right)^{2}\left[1+\frac{3}{\pi \sqrt{a}} \operatorname{coth}(\pi \sqrt{a})-\frac{6}{\pi} \sqrt{\frac{2}{a}} \operatorname{coth}\left(\pi \sqrt{\frac{a}{2}}\right)+\frac{9}{\pi^{2} a}\right]\right\}
\end{aligned}
$$

The mean squared end-to-end distance, $\left\langle\mathbf{R}^{2}\right\rangle=\left\langle\mathbf{X}^{2}\right\rangle+\left\langle\mathbf{Y}^{2}\right\rangle+\left\langle\mathbf{Z}^{2}\right\rangle$ (where $\mathbf{R}=\mathbf{r}_{N}-\mathbf{r}_{1}$ ), is obtained in a similar manner

$$
\left\langle\mathbf{X}^{2}\right\rangle=\frac{N b^{2}}{3}\left[1+\left(\dot{\gamma} \tau_{1}\right)^{2} \frac{8}{\pi^{2}} \sum_{p=1 \text { :odd }}^{\infty} \frac{1}{p^{2}\left(a+p^{2}\right)\left(a+2 p^{2}\right)}\right]
$$




$$
\begin{aligned}
& =\frac{N b^{2}}{3}\left\{1+\left(\frac{\dot{\gamma} \tau_{1}}{a}\right)^{2}\left[1+\frac{2}{\pi \sqrt{a}} \tanh \left(\frac{\pi \sqrt{a}}{2}\right)-\frac{4}{\pi} \sqrt{\frac{2}{a}} \tanh \left(\frac{\pi}{2} \sqrt{\frac{a}{2}}\right)\right]\right\} \\
\left\langle\mathbf{Y}^{2}\right\rangle & =\frac{N b^{2}}{3} \\
\left\langle\mathbf{Z}^{2}\right\rangle & =\frac{N b^{2}}{3} \frac{2}{\pi \sqrt{a}} \tanh \left(\frac{\pi \sqrt{a}}{2}\right)
\end{aligned}
$$

where the three analytical summations are down following Ref. ${ }^{28}$ Setting $a=0$, we obtain the limiting case first found by Bhattacharyya and Cherayil ${ }^{29}$ where Eq. (19) simplifies to $\left\langle\mathbf{X}^{2}\right\rangle=\frac{N b^{2}}{3}\left[1+\frac{\pi^{4}}{240}\left(\dot{\gamma} \tau_{1}\right)^{2}\right]$. On the other hand for $\dot{\gamma}=0$, only the $Z$ component is squeezed and the $X$ and $Y$ components remain unaffected, a classical result for Gaussian polymers. ${ }^{30}$

The eigenvalues of the shape tensor are $\left(\lambda_{+}, I_{y y}, \lambda_{-}\right)$where

$$
\lambda_{ \pm}=\frac{1}{2}\left(I_{x x}+I_{z z}\right) \pm \frac{1}{2} \sqrt{\left(I_{x x}-I_{z z}\right)^{2}+4 I_{x z}^{2}}
$$

The eigenvectors are $\left(\mathbf{e}_{+}, \mathbf{e}_{y}, \mathbf{e}_{-}\right)$where $\mathbf{e}_{+}=\cos \Theta \mathbf{e}_{x}+\sin \Theta \mathbf{e}_{z}$ and $\mathbf{e}_{-}=-\sin \Theta \mathbf{e}_{x}+\cos \Theta \mathbf{e}_{z}$ with $\cos \Theta=\left(\lambda_{+}-I_{z z}\right) / \sqrt{I_{x z}^{2}+\left(\lambda_{+}-I_{z z}\right)^{2}}$. The measured aspect ratio in the $(x, y)$ plane, also called anisotropy of the molecule, is:

$$
\frac{R_{\max }}{R_{\min }}=\sqrt{\frac{\lambda_{+}}{I_{y y}}} \cos \Theta
$$

For $a \gg 1$, we can use the asymptotic behaviours for the shape tensor components

$$
I_{z z} \simeq \frac{N b^{2}}{6 \pi \sqrt{a}} \quad ; \quad I_{x z} \simeq N b^{2} \frac{\dot{\gamma} \tau_{1}}{a^{3 / 2}} \frac{\sqrt{2}-1}{6 \pi} \quad ; \quad I_{x x} \simeq \frac{N b^{2}}{18}\left[1+\left(\frac{\dot{\gamma} \tau_{1}}{a}\right)^{2}\right]
$$

and the end-to-end distance coordinates

$$
\left\langle\mathbf{X}^{2}\right\rangle \simeq \frac{N b^{2}}{3}\left[1+\left(\frac{\dot{\gamma} \tau_{1}}{a}\right)^{2}\right] \quad ; \quad\left\langle\mathbf{Z}^{2}\right\rangle \simeq \frac{2 N b^{2}}{3 \pi \sqrt{a}}=\frac{2}{\sqrt{3 \pi}} b \sqrt{\frac{k_{\mathrm{B}} T}{\Gamma}}
$$

We have checked that as soon as $a \geq 100$ or $W i=\dot{\gamma} \tau_{1} \geq 100$, an extremely good approxi- 


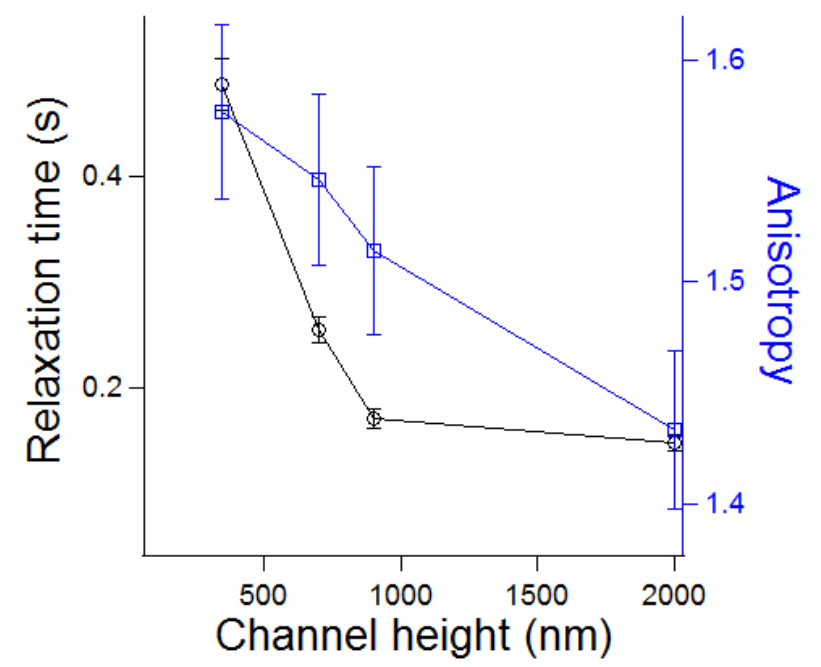

Figure 2: Relaxation time (black dataset) and anisotropy (blue dataset) as a function of the channel height for $\lambda$-DNA molecules without hydrodynamic nor electrophoretic actuation.

mation is:

$$
\frac{R_{\max }}{R_{\min }} \simeq \sqrt{\frac{I_{x x}}{I_{y y}}} \simeq \sqrt{\frac{\left\langle\mathbf{X}^{2}\right\rangle}{\left\langle\mathbf{Y}^{2}\right\rangle}} \simeq\left[1+\left(\frac{\dot{\gamma} \tau_{1}}{a}\right)^{2}\right]^{1 / 2}
$$

\section{Results and discussion}

\section{Relaxation time and conformation at rest}

We first monitored the conformation of DNA in the different channels of height $h=2.0,0.9$, 0.7, $0.4 \mu \mathrm{m}$ at equilibrium with no force field. We observed an increase of the relaxation time with the degree of confinement from $\tau_{0}=0.15$ to $0.5 \mathrm{~s}$ (Fig. 2) associated to an onset in anisotropy of the molecule from 1.43 to 1.58 . These trends have been observed and thoroughly analyzed in Refs. ${ }^{21,22}$ Interestingly, these values of the anisotropy imply that excluded volume effects cannot be overlooked, and the variation of the relaxation time with the channel height suggests that hydrodynamic interactions are not negligible. However, in the case where the flow and electric fields are actuated, the DNA is localized close to the channel walls and hydrodynamic interactions are likely screened out. 


\section{DNA conformation with electro-hydrodynamic actuation}

For a channel height of $2.0 \mu \mathrm{m}$, we measured the major and minor axis $\left(R_{\max }, R_{\min }\right)$ for 10 molecules at least for each experimental setting in pressure and voltage, each molecule being imaged during 50 to 500 consecutive frames. The blue dataset of Fig. 3A reports the average major radius as a function of the electrophoretic velocity, $V_{E}$. The major axis $R_{\max }$ drops from $3.9 \mu \mathrm{m}$ for flowing molecules to a saturation value of $1.0 \mu \mathrm{m}$ as the molecules are roughly stopped by the electric field (crossing between the red curve and black dashed line in Fig. 3A). This contraction is also associated to the gradual narrowing of the major axis distribution (Fig. 3B), indicating that length fluctuations tend to decrease for increasing transverse visco-elastic forces toward the channel walls.

We then focus on electro-hydrodynamic actuation conditions that correspond to DNA null velocity. Looking at Fig. 3A, we first note that molecules are halted for an electrophoretic velocity $V_{E}=43 \mu \mathrm{m} / \mathrm{s}$, which is much smaller than the hydrodynamic velocity $V_{0}=290 \mu \mathrm{m} / \mathrm{s}$. This difference is accounted for by the occurrence of transverse viscoelastic forces oriented toward the walls. ${ }^{16}$ More quantitatively, the equality between the electrophoretic and hydrodynamic velocities defines an effective distance from the walls of $V_{E} / \dot{\gamma} \approx 75 \mathrm{~nm}$, which is an order of magnitude less than the radius of gyration of $\lambda$-DNA in bulk of $0.73 \mu \mathrm{m} .{ }^{31}$ As shown by Smith et al., ${ }^{31}$ in their experiments, $\lambda$-DNA is indeed in good solvent conditions with an excluded volume on the order of $v \simeq 0.2 b^{3}$. Using Boltzmann statistics with the force expression in Eq. (2), we can compute the average vertical position $\langle z\rangle \sim\left(V_{E} V_{0}\right)^{-1 / 2}$. This expression can be combined with the balance between the electrophoretic and hydrodynamic velocity to deduce that the conditions of arrest are associated to $V_{E} \sim V_{0}^{1 / 3}$. This prediction, which is plotted as a dashed in line Fig. 3C, is consistent with our measurements of the electric vs. hydrodynamic velocities conditions of arrest, although the power-law exponent for the best fit is 0.41 (solid line in Fig. 3C). 

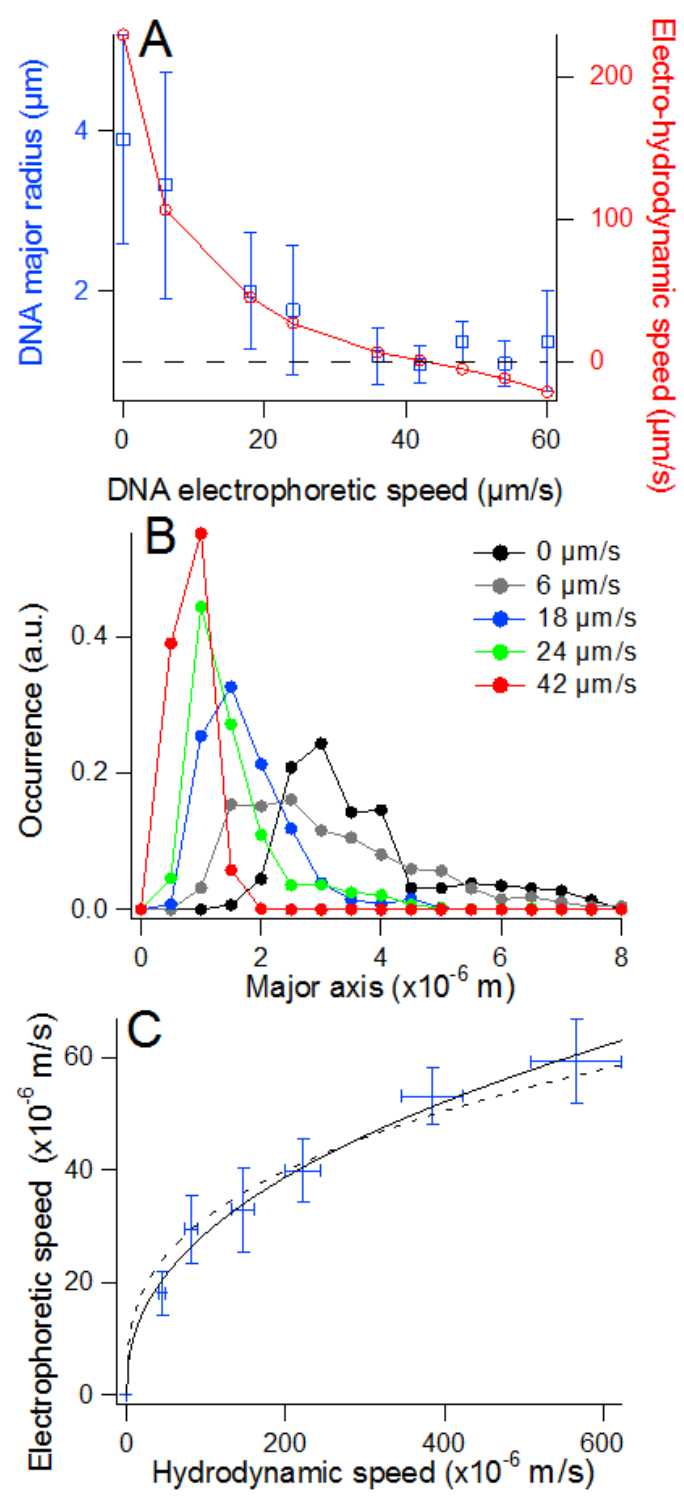

Figure 3: DNA conformation and velocity with electro-hydrodynamic actuation $(h=2 \mu \mathrm{m})$ : (A) Variation of the major axis $R_{\max }$ and migration velocity as a function of the electrophoretic velocity $V_{E}$ (blue and red datasets, respectively). The flow velocity is $V_{0}=$ $290 \mu \mathrm{m} / \mathrm{s}(W i \simeq 80)$. The black dashed line corresponds to DNA null velocity. (B) $R_{\max }$ distribution for 5 different $V_{E}$ values. (C) The blue dataset presents 6 different conditions of electrophoretic vs. hydrodynamic velocities associated to DNA null velocity $(20 \leq W i \leq 200$ for $h=2 \mu \mathrm{m})$. The black dashed line is the fit with our model ${ }^{19}$ of transverse viscoelastic force oriented toward the walls, $V_{E} \sim V_{0}^{1 / 3}$. The black solid line is a fit with a free exponent equal to 0.41 .

\section{Comparison to the model}

We first assume that DNA is a Gaussian chain to quantitatively compare our experimental results to the analytical Rouse model. The elastic constant characterizing polymer segments 
$k \simeq 10^{-3} \mathrm{pN} / \mathrm{nm}$, the Rouse time $\tau_{1}=19.5 \mathrm{~s}$, and the viscoelastic transverse force $\Gamma \simeq$ $1.6 \times 10^{-5}\left(V_{0} V_{E}\right) / h^{2} \mathrm{pN} / \mathrm{nm}$ lead us to estimate that $a=\tau_{1} \Gamma / \xi$ is between 5000 and 20000 in our experimental settings. Therefore, the use of the asymptotic formulas Eq. (26) is justified.

Because $a \propto \Gamma \propto V_{0} V_{E}$ and $\dot{\gamma} \propto V_{0}$, we deduce from Eq. (26) that the anisotropy is strongly dependent on the electrophoretic velocity, and that it decreases to 1 as the electrophoretic velocity increases. This trend is essentially due to cross-correlation between the polymer dynamics in the $z$ and $x$ directions, as developed in Eq. (13). We thus plotted the anisotropy of the molecule for $V_{0}=290 \mu \mathrm{m} / \mathrm{s}(W i \simeq 80)$ for a range of values of the electrophoretic velocity $V_{E}$ and fitted this data based on Eq. (26) (Fig. 4A). The fit required that we introduce a coefficient $\gamma$ in front of $\Gamma$ equal to 0.05 . This correction is equivalent to defining an effective electrophoretic velocity much smaller than the real one. This prefactor reduces the amplitude of transverse viscoelastic forces, setting the molecule in a more anisotropic configuration. Its addition is required because we neglect volume exclusion between monomers in our model and hence predict that the anisotropy should drop to 1 , whereas this parameter remains greater than 1.3 in our experiments. Note that this value of the anisotropy of 1.3 or more has been described in several reports of the literature. ${ }^{21}$ Finally, we remark that the reduced apparent mobility shows some analogy with the prediction that polyelectrolytes deformed by the action of a flow may have a slower electrophoretic migration when compared to the expectations for a random coil DNA. ${ }^{32}$

We tested the model further by analyzing the anisotropy of the molecules in experimental conditions of null velocity, which correspond to $V_{E} \sim V_{0}^{1 / 3}$ and for a channel height of $0.9 \mu \mathrm{m}$. In this case also, the fit of the anisotropy as a function of the maximal flow velocity $V_{0}$ is consistent with our model (Fig. 4B), provided that we consider the same correction factor in front of $\Gamma$ of 0.052 .

Altogether, we thus conclude that the polymer model is in qualitative agreement with our data. It suggests that, as the flow velocity increases, the DNA molecules should progressively 

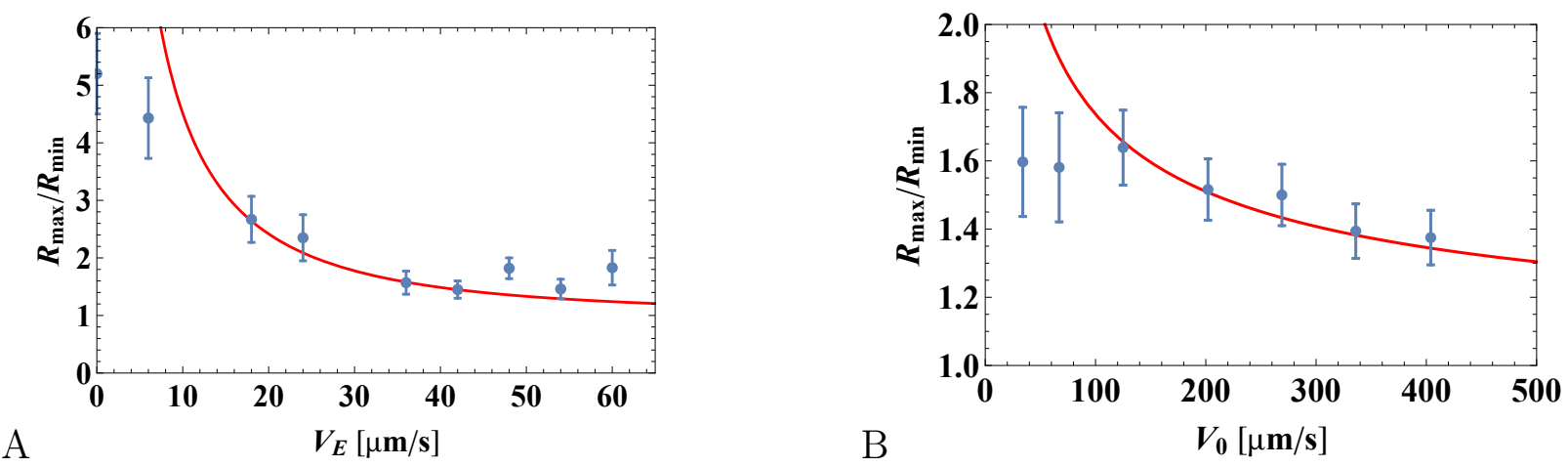

Figure 4: (A) DNA Anisotropy $R_{\max } / R_{\min }$ vs. the electrophoretic velocity $V_{E}$ and the corresponding fit (red solid line) with our model using $\Gamma=0.4 \times 10^{-8} V_{0} V_{E} \mathrm{~N} / \mathrm{m}(h=2 \mu \mathrm{m})$, $b=0.1 \mu \mathrm{m}$ and a fitting parameter $\gamma=0.054$ in front of $\Gamma$. (B) Same as in (A), $R_{\max } / R_{\min }$ vs. the flow velocity $V_{0}$ assuming $V_{E}=43\left(V_{0} / 280\right)^{1 / 3} \mu \mathrm{m} / \mathrm{s}$ (see Fig. 3C), $\tau_{1}=19.5 \mathrm{~s}$ $(L=21.1 \mu \mathrm{m}, b=0.1 \mu \mathrm{m}), h=0.9 \mu \mathrm{m}$ and $\gamma=0.052$.

contract and reach a conformation resembling that of a confined pancake-like equilibrium configuration.

Using the fitted value for $\Gamma_{\text {fit }}=\gamma \Gamma$, we are able to check if our assumption of the neglect of the interaction between the DNA and the wall is valid. Using the asymptotic formula, Eq. (24), and assuming $V_{E}=43\left(V_{0} / 280\right)^{1 / 3} \mu \mathrm{m} / \mathrm{s}$ for $h=2 \mu \mathrm{m}$, we obtain for the typical DNA thickness in the direction perpendicular to the walls, $\sqrt{I_{z z}}=167 V_{0}^{-1 / 3} \mathrm{~nm}$ (where $V_{0}$ is expressed in $\mu \mathrm{m} / \mathrm{s}$ ). Hence for the experimental values $50 \leq V_{0} \leq 400 \mu \mathrm{m} / \mathrm{s}$, we find that $10 \leq \sqrt{I_{z z}} \leq 50 \mathrm{~nm}$. Comparing to the typical distance of the DNA center of mass, $\langle z\rangle=\sqrt{\frac{2 k_{\mathrm{B}} T}{\pi \Gamma_{\mathrm{fit}}}}, 19$ we find $0.45 \leq \frac{\sqrt{I_{z z}}}{\langle z\rangle} \leq 1$, which confirms that contacts between the DNA and the wall are sparse and can be neglected in a first approximation. The presence of the wall should play a role on the DNA conformation for smaller $h$ and high flow velocities $V_{0} \simeq 400 \mu \mathrm{m} / \mathrm{s}$, but a detailed model should consider the electrostatic repulsion between the DNA and the wall, which is beyond the scope of this study. Moreover the polymer in-plane radius of gyration, defined as $R_{G \|}=\left(I_{x x}+I_{y y}\right)^{1 / 2}$, is on the order of $1 \mu \mathrm{m}$ and is therefore much larger than $\sqrt{I_{z z}}$ and $\langle z\rangle$. Hence most of the monomers are located in vicinity of the wall thus justifying our assumption of the neglect of hydrodynamics interactions. 


\section{Distribution of major and minor axis}

In order to clarify the conformation of DNA molecules in electro-hydrodynamic viscoelastic flows, we extracted the size distribution of the major and minor axis in conditions of null velocity. Setting the channel height to $0.9 \mu \mathrm{m}$, the distributions are plotted for a range of Weissenberg number in Fig. 5A. Alternatively, the distributions can be compared at a given Weissenberg number, $W i=300$, and with three channel heights spanning 0.4 to 2.0 $\mu \mathrm{m}$ (Fig. 5B). Each distribution is plotted with points superimposed with the fit derived from the 2D polymer chain model in good solvent, $P(x)=P\left(x_{0}\right) \exp \left(-\frac{\kappa}{2}\left[\left(\frac{x_{0}}{x}\right)^{4}+\left(\frac{x}{x_{0}}\right)^{4}-2\right]\right)$ with $x$ the radius of gyration and $x_{0}$ the value for which the maximum probability occurs (solid lines in Fig. 5). ${ }^{33}$ Note that the normalization of the distribution defines a relationship between its amplitude $P\left(x_{0}\right)$ and the parameter $\kappa$ that controls its width. Using numerical approximations for $x_{0}$ and $\kappa$ close to unity, we obtained $P\left(x_{0}\right)^{-1}=x_{0}\left(0.7554 / \kappa^{1 / 3}-0.1686\right)$.

First, focusing on the data for a constant channel height of $0.9 \mu \mathrm{m}$ (Fig. 5A), the plots show an offset between the minor and major axis peaks associated to an anisotropy of $\simeq 1.5$, which is comparable to the value of $\simeq 1.4$ for confined DNA in $2 \mathrm{D}$ slit-like geometry with a channel height of $50 \mathrm{~nm} .{ }^{21}$ Furthermore, the minor axis distribution is expected to be consistent with a polymer chain at rest. Indeed, the average position of its peak at $0.63 \pm 0.05 \mu \mathrm{m}$ is comparable to $\lambda$-DNA radius of gyration at rest $R_{g} \simeq 0.73 \mu \mathrm{m} .{ }^{31}$ The shape of this distribution is also consistent with the response for an equilibrium 2D polymer chain. The distribution of the major axis instead appears to be broader and skewed toward longer sizes in comparison to the distribution of minor axis (lower vs. upper panels in Fig. 5A). Its shape departs from the equilibrium polymer model due to the occurrence of extended configurations, that appear to be particularly marked for low Weissenberg numbers. This result is consistent with the prediction of our model that the increase of the flow velocity progressively confines the chain in a conformation resembling that of an equilibrium chain (red dataset in Fig. 5A). 

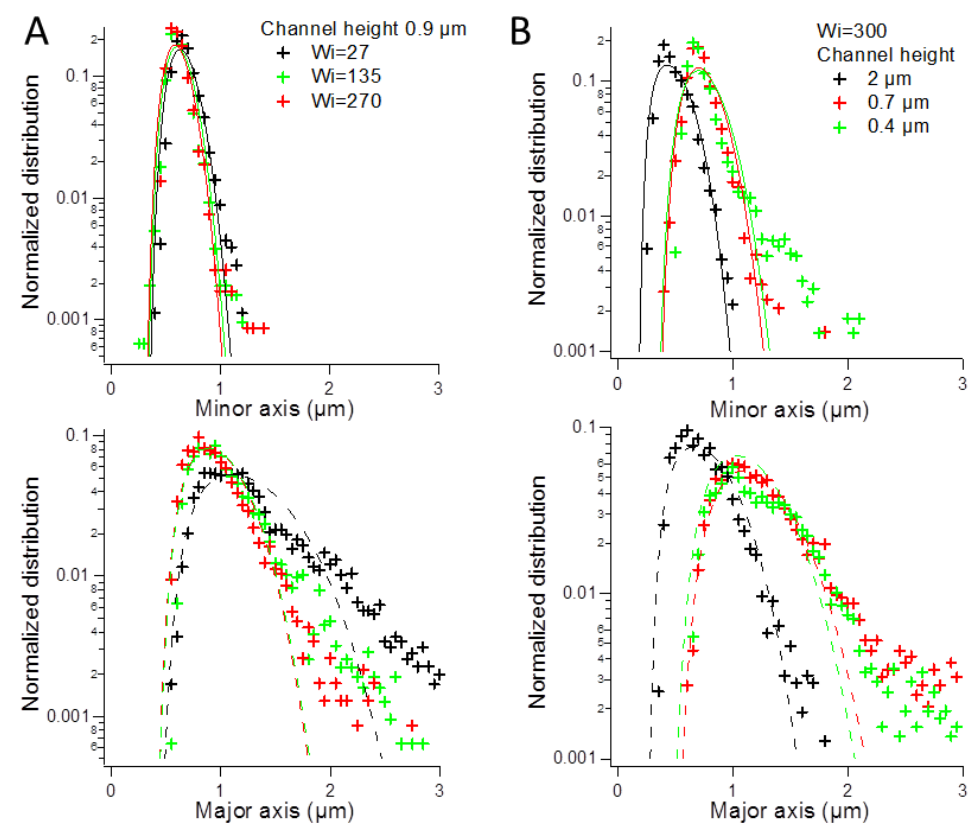

Figure 5: Distributions of minor $R_{\min }$ (upper panel) and major $R_{\max }$ (lower panel) axis distribution at null velocity: (A) for different Weissenberg numbers $W i$ at constant channel height $h=0.9 \mu \mathrm{m}$. Solid lines correspond to fits with a 2D polymer chain model (see text). Each histogram is obtained from at least 8 molecules tracked during 100 consecutive images or more, meaning that they include 2,000 to 4,000 measurements. (B) Same as in (A) for a given Weissenberg number of $W i=300$ and three different channel heights $h$.

Second, the analysis of the distributions for different channel heights at high Wi $\simeq 300$ (Fig. 5B) indicates the good agreement of the equilibrium 2D polymer model for the minor and major axis at $h=2 \mu \mathrm{m}$, but also that the minor axis distribution is not fitted with the 2D polymer model at equilibrium for high levels of confinement of $0.4 \mu \mathrm{m}$. It appears that events characterized by large deformations become more frequent.

\section{DNA tumbling}

Intriguingly, the polymer dynamics represented by the major to minor axis over time appears to be non-stationary with many events where the polymer extends in the direction of the flow and then retracts before its extension builds up again (Fig. 6A). These dynamics are reported in a plot of the anisotropy of the molecule over time in Fig. 6B. Whereas the molecule shape 

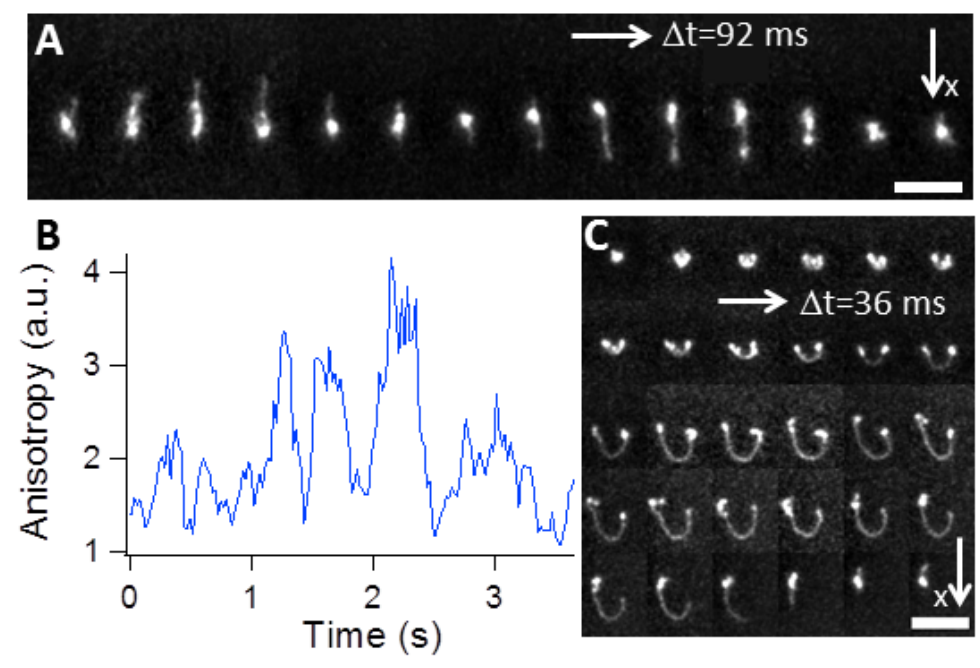

Figure 6: Evidence of intermittent fluctuations: (A) Time series of a $\lambda$-DNA molecule flowing in a channel of $h=2 \mu \mathrm{m}$ at a Weissenberg number $W i=15$ and an electrophoretic velocity $V_{E}=15 \mu \mathrm{m} / \mathrm{s}$ ( $\Delta t$ is the laps time between successive images). (B) The plot shows the anisotropy vs. time for the molecule recorded in (A). (C) Time series of a $\lambda$-DNA molecule flowing in a shallower channel $\left(h=0.4 \mu \mathrm{m}, W i=210, V_{E}=42 \mu \mathrm{m} / \mathrm{s}\right)$.

remained elliptical and oriented by the flow for low levels of confinement, extension events in channels of $0.4 \mu \mathrm{m}$ appeared to last during longer period and to be associated to a catenary shape (Fig. 6C). We suggest that the occurrence of such events biases the analysis of the minor axis distribution with 2D polymer models, likely accounting for the anomalous shape reported in the upper panel of Fig. 5B.

In order to clarify these dynamics, we report the autocorrelation function of the tilt angle of the molecule

$$
A C F(\tau)=\frac{1}{i_{\max }-s} \sum_{i=0}^{i_{\max }-s-1} \theta(i \Delta t) \theta(i \Delta t+\tau)
$$

where $\Delta t$ is the time interval and $\tau$ the temporal variable. Using a mono-exponential curve fitting (solid line in the inset of Fig. 7), we estimate the slowest relaxation time $\tau_{R}$. The Rouse time of the fluctuations obtained without electro-hydrodynamic actuation $\tau_{0} \simeq 0.5 \mathrm{~s}$ is roughly 6 times greater than $\tau_{R}$ at flow velocities of $200 \mu \mathrm{m} / \mathrm{s}$. These dynamics are therefore not controlled by this Rouse time nor by the fast relaxation time in the $z$ direction $\tau_{\Gamma}=\xi / \Gamma$. In Fig. $7, \tau_{R}$ is plotted as a function of $V_{0}$ for five different channel heights spanning 0.4 


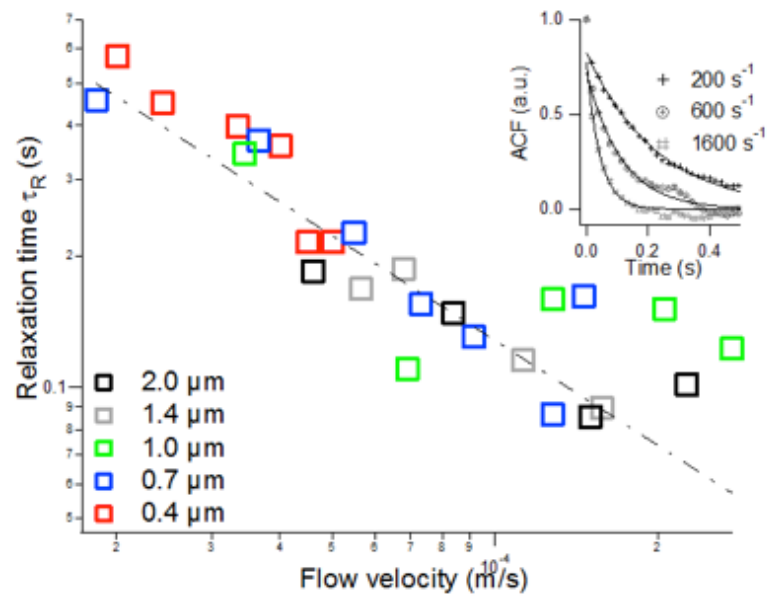

Figure 7: Rotational relaxation dynamics of $\lambda$-DNA molecules. Relaxation time $\tau_{R}$ plotted as a function of the flow velocity $V_{0}$ for different channel heights. The dashed line is the power law with an exponent equal to -0.83 . In the inset, three time autocorrelation functions (ACF) of the tilt angle $\theta$, as defined in Fig. 1C, are shown for different shear rates in a channel of $0.7 \mu \mathrm{m}$. The lines correspond to fits with mono-exponential functions.

to $2.0 \mu \mathrm{m}$. The relaxation time $\tau_{R}$ appears to depend on flow velocity (Fig. 7) following a power law exponent of $-0.83 \pm 0.07$. This dependence is reminiscent of measurements and simulations for tumbling dynamics in bulk flows in the range $-2 / 3$ to $-3 / 4 .^{7,34,35}$ More precisely, the exponent we measured of $-0.83 \pm 0.07$ appears to be in qualitative agreement with that of $-3 / 4$ which corresponds to the case of a Gaussian chain with no hydrodynamic interaction. ${ }^{35}$ This qualitative agreement tends to confirm our hypothesis that hydrodynamic interactions are screened out near the walls. We thus suggest that the time $\tau_{R}$ is controlled by the existence of intermittency phenomena as described for tumbling events in Poiseuille flows. ${ }^{7}$ Yet, the fact that these intermittent dynamics appear to be independent of the channel height $h$ in our experimental settings remains unclear.

In order to clarify the molecular origin of these tumbling events, we finally performed Brownian dynamics simulations using a bead-spring model derived from Eq. (3). We assigned the amplitude of the prefactor of the transverse force in Eq. (2) to 0.4 using our calibration in ref., ${ }^{19}$ and the simulation time step to $1 \mathrm{~ms}$. The molecule migration was stalled for $\left(V_{0}, V_{E}\right)$ equal to $(50,12) \mu \mathrm{m} / \mathrm{s}$, in agreement with our measurements of $(50,15) \mu \mathrm{m} / \mathrm{s}$ (see 

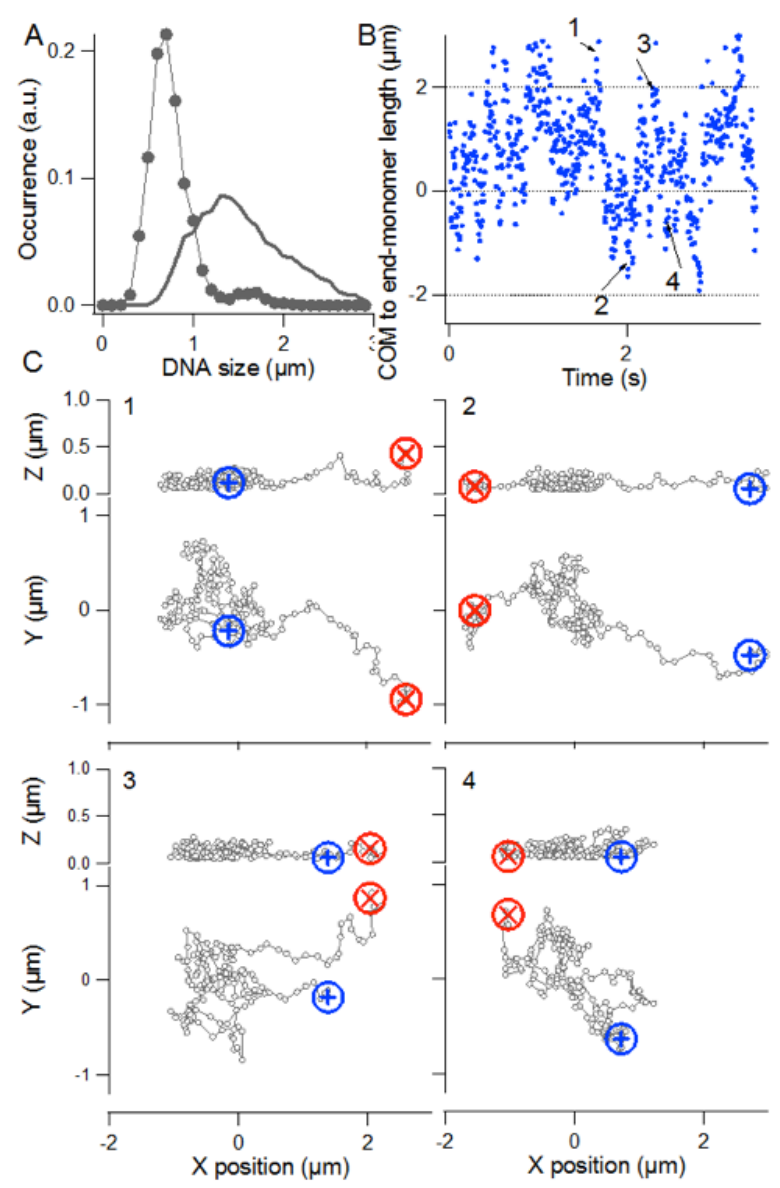

Figure 8: Results from Brownian dynamics simulations. (A) The size distributions of the major (line) and minor (circles) axis are shown for a flow velocity $V_{0}=50 \mu \mathrm{m} / \mathrm{s}$ and an electrophoretic velocity $V_{E}=15 \mu \mathrm{m} / \mathrm{s}$. (B) Distance between the molecule center of mass $(\mathrm{COM})$ and the red end monomer as a function of time. (C) The four panels represent snapshots of the conformation of the molecule at the time steps tagged in panel (B). The end monomers are marked with blue and red circles.

Fig. 3C). For arrested molecules, the conformation was characterized by an anisotropy of $\sim 2.0$ (Fig. $8 \mathrm{~A}$ ), that was slightly larger than $\sim 1.75$ in our experiments. Furthermore the analysis of the intermittent fluctuations in the $x$ axis (Fig. 8B) indicated that the contraction of the molecule was associated to spooling around its stagnation position. Note that the amplitude of tumbling events observed in Ref. ${ }^{6}$ is much larger than in our case, likely because the molecule remains contracted in the flow direction. Continuous recirculation of the end monomers around the bulk of the chain, which are marked in red and blue in Fig. 8C, 
is detected. The cyclic motion starts with the fluctuation of one monomer away from the wall. Its position is then higher than the center of mass of the molecule, and this fluctuation is converted into a displacement along the $x$-direction by the shear of the flow. The monomer is simultaneously dragged back toward the surface by the viscoelastic force. Conversely, the occurrence of vertical fluctuation towards the surface brings the monomer in a region dominated by electrophoresis, so the longitudinal displacement is oriented oppositely. Interestingly, this cyclic motion is reminiscent of the anomalous dynamics of end-tethered polymer chains, ${ }^{36}$ which undergo continual recirculation around their stagnation position. We note the fact that end-monomer can travel forward or backward from the bulk of the molecule can be clearly detected in the montage reported in Fig. 6A.

\section{Conclusion}

In conclusion, we performed a thorough experimental and theoretical investigation of DNA response in shear flows combined to electrophoretic actuation under viscoelastic confinement. In contrast to bulk shear flows, which extend the molecule, DNA appears to adopt a much more contracted conformation. Contraction appears to be associated to intermittent fluctuations reminiscent of tumbling events, which are determined by the velocity of the flow and associated to the winding of the molecule about its center of mass. Because shear flows can lead to accumulation of elastic energy, ${ }^{5}$ we suggest that this spooling mechanism minimizes the storage of elastic energy along the contour of the chain.

In the analytical model, we have neglected both the swelling of the DNA due to excluded volume and electrostatic interactions and the influence of hydrodynamics interactions on DNA dynamics. These issues are of course not tractable analytically and should be tackled numerically through, for instance, Brownian dynamics simulations that include hydrodynamics interactions by using the Rotne-Prager tensor and hydrodynamic images associated to the presence of the confining surfaces, ${ }^{37}$ or through multiparticle collision dynamics tech- 
niques that have been recently applied to the case of ring polymers in shear flows ${ }^{38}$ and polyelectrolytes in salt-free solutions. ${ }^{39}$

A direct technological application of DNA transport in viscoelastic flows with electrohydrodynamic actuation is DNA separation and concentration. ${ }^{16,19,40}$ This assay showed good separation performances for molecules in the size range 0.1 to $10 \mathrm{kbp}$. We thus suggest that better models of DNA transport could have strong impact for the manipulation of molecules of higher molecular weight, $100 \mathrm{kbp}$ or more. In this size range, the number of technologies for DNA analysis and processing is limited, and the remaining method of choice since the early 80 's is pulsed-field gel electrophoresis despite its constraints in labor and time. ${ }^{41}$

\section{Acknowledgement}

The authors thank the LAAS-CNRS technology platform, member of the French network Renatech, for their technical support. This work was partly supported by the Midi-Pyrénées grant "Gene-Extractor" and the ANR grant $\mu \mathrm{LAS}$ (ANR-16-CE18-0028-01). B.C. thanks the Ligue Contre le Cancer for her Ph.D. fellowship. H.R. and A.L. thank the French ministry of research for their PhD fellowships. M.M. thanks H. Watanabe for pointing us the analytical expressions of the summations in Eqs. (17)-(19).

\section{References}

(1) Lumley, J. L. Drag reduction by additives. Ann. Rev. Fluid Mech. 1969, 1, 367-384.

(2) de Gennes, P. G. Coil-stretch transition of dilute flexible polymers under ultrahigh velocity gradients. J. Chem. Phys. 1974, 60, 5030-5042.

(3) Perkins, T. T.; Smith, D. E.; Chu, S. Single Polymer Dynamics in an Elongational Flow. Science 1997, 276, 2016-2021. 
(4) Smith, D. E.; Babcock, H. P.; Chu, S. Single-Polymer Dynamics in Steady Shear Flow. Science 1999, 283, 1724-1727.

(5) Teixeira, R. E.; Babcock, H. P.; Shaqfeh, E. S.; Chu, S. Shear Thinning and Tumbling Dynamics of Single Polymers in the Flow-Gradient Plane. Macromolecules 2005, 38, 581592.

(6) Schroeder, C. M.; Teixeira, R. E.; Shaqfeh, E. S.; Chu, S. Characteristic Periodic Motion of Polymers in Shear Flow. Phys. Rev. Lett. 2005, 95, 18301.

(7) Gerashchenko, S.; Steinberg, V. Statistics of Tumbling of a Single Polymer Molecule in Shear Flow. Phys. Rev. Lett. 2006, 96, 38304.

(8) Winkler, R. G. Semiflexible Polymers in Shear Flow. Phys. Rev. Lett. 2006, 97, 128301.

(9) Agarwal, U. S.; Dutta, A.; Mashelkar, R. A. Migration of Macromolecules under Flow: The Physical Origin and Engineering Implications. Chem. Eng. Sc. 1994, 49, 16931717.

(10) Jendrejack, R. M.; Dimalanta, E. T.; Schwartz, D. C.; Graham, M. D.; de Pablo, J. J. DNA Dynamics in a Microchannel. Phys. Rev. Lett. 2003, 91, 038102.

(11) Ma, H.; Graham, M. D. Theory of Shear-Induced Migration in Dilute Polymer Solutions near Solid Boundaries. Phys. Fluids 2005, 17, 83103.

(12) Usta, O. B.; Butler, J. E.; Ladd, A. Theory of Shear-Induced Migration in Dilute Polymer Solutions near Solid Boundaries. Phys. Fluids 2006, 18, 31703.

(13) Graham, M. D. Fluid Dynamics of Dissolved Polymer Molecules in Confined Geometries. Ann. Rev. Fluid Mech. 2011, 43, 273-298.

(14) Cannavacciuolo, L.; Winkler, R. G.; Gompper, G. Mesoscale Simulations of Polymer Dynamics in Microchannel Flows. EPL (Europhysics Letters) 2008, 83, 34007. 
(15) He, Q.; Ranchon, H.; Carrivain, P.; Viero, Y.; Lacroix, J.; Darran, E.; Victor, J. M.; Bancaud, A. Hydrodynamic Conformational Manipulation of DNA in Nanochannels. Macromolecules 2013, 46, 6195-6202.

(16) Ranchon, H.; Malbec, R.; Picot, V.; Boutonnet, A.; Terrapanich, P.; Joseph, P.; Leichle, T.; Bancaud, A. DNA Separation and Enrichment Using Electro-Hydrodynamic Bidirectional Flows in Viscoelastic Liquids. Lab Chip 2016, 16, 1243-1253.

(17) Günther, K.; Mertig, M.; Seidel, R. Mechanical and structural properties of YOYO-1 complexed DNA. Nucl. Acids Res. 2010, 38, 6526-6532.

(18) McFarlane, N.; Wagner, N.; EW, E. K.; Lynch, M. Poly(ethylene oxide) (PEO) and poly(vinyl pyrolidone) (PVP) induce different changes in the colloid stability of nanoparticles. Langmuir 2010, 26, 13823-13830.

(19) Chami, B.; Socol, M.; Manghi, M.; Bancaud, A. Modeling of DNA Transport in Viscoelastic Electro-Hydrodynamic Flows for Enhanced Size Separation. Soft Matter 2018, $14,5069-5079$.

(20) Ranchon, H.; Picot, V.; Bancaud, A. Metrology of Confined Flows Using Wide Field Nanoparticle Velocimetry. Sci. Rep. 2015, 5, 10128.

(21) Bonthuis, D. J.; Meyer, C.; Stein, D.; Dekker, C. Conformation and Dynamics of DNA Confined in Slitlike Nanofluidic Channels. Phys. Rev. Lett. 2008, 101, 108303.

(22) Hsieh, C.-C.; Balducci, A.; Doyle, P. An experimental study of DNA rotational relaxation time in nanoslits. Macromolecules 2007, 40, 5196-5205.

(23) Leshansky, A. M.; Bransky, A.; Korin, N.; Dinnar, U. Tunable Nonlinear Viscoelastic "Focusing" in a Microfluidic Device. Phys. Rev. Lett. 2007, 98, 234501.

(24) Seo, K. W.; Byeo, H. J.; Huh, H. K.; Lee, S. J. Particle migration and single-line particle focusing in microscale pipe flow of viscoelastic fluids. $R S C A d v$. 2014, 4, 3512-3520. 
(25) Doi, M.; Edwards, S. F. The Theory of Polymer Dynamics; Oxford University Press Inc.: New York, 1986.

(26) Brunet, A.; Tardin, C.; Salomé, L.; Rousseau, P.; Destainville, N.; Manghi, M. Dependence of DNA Persistence Length on Ionic Strength of Solutions with Monovalent and Divalent Salts: A Joint Theory-Experiment Study. Macromolecules 2015, 48, 36413652.

(27) Doi, M.; Nakajima, H. Theory of the Shape Relaxation of a Rouse Chain. Chem. Phys. $\mathbf{1 9 7 4}, 6,124-129$.

(28) Watanabe, H.; Matsumiya, Y. Viscoelastic and Dielectric Relaxation of Reptating Type-A Chains Affected by Reversible Head-to-Head Association and Dissociation. Macromolecules 2018, 51, 6476-6496.

(29) Bhattacharyya, P.; Cherayil, B. Chain extension of a confined polymer in steady shear flow. J. Chem. Phys. 2012, 137, 194906.

(30) de Gennes, P.-G. Scaling concepts in polymer physics; Cornell University Press: New York, 1979.

(31) Smith, D. E.; Perkins, T. T.; Chu, S. Dynamical Scaling of DNA Diffusion Coefficients. Macromolecules 1996, 29, 1372-1373.

(32) Long, D.; Viovy, J.-L.; Ajdari, A. Simultaneous action of electric fields and nonelectric forces on a polyelectrolyte: motion and deformation. Phys. Rev. Lett. 1996, 76, 3858.

(33) Victor, J.-M.; Lhuillier, D. The Gyration Radius Distribution of Two-Dimensional Polymer Chains in a Good Solvent. J. Chem. Phys. 1990, 92, 1362-1364.

(34) Usabiaga, F. B.; Delgado-Buscalioni, R. Characteristic Times of Polymer Tumbling under Shear Flow. Macromol. Theor. Simul. 2011, 20, 466-471. 
(35) Dalal, I. S.; Albaugh, A.; Hoda, N.; Larson, R. G. Tumbling and Deformation of Isolated Polymer Chains in Shearing Flow. Macromolecules 2012, 45, 9493-9499.

(36) Doyle, P. S.; Ladoux, B.; Viovy, J. L. Dynamics of a Tethered Polymer in Shear Flow. Phys. Rev. Lett. 2000, 84, 4769-4772.

(37) Manghi, M.; Schlagberger, X.; Kim, Y.-W.; Netz, R. R. Hydrodynamic effects in driven soft matter. Soft Matter 2006, 2, 653.

(38) Chen, W.; Zhao, H.; Liu, L.; Chen, J.; Li, Y.; An, L. Effects of excluded volume and hydrodynamic interaction on the deformation, orientation and motion of ring polymers in shear flow. Soft Matter 2015, 11, 5265-5273.

(39) Liu, L.; Chen, W.; Chen, J. Shape and Diffusion of Circular Polyelectrolytes in Salt-Free Dilute Solutions and Comparison with Linear Polyelectrolytes. Macromolecules 2017, $50,6659-6667$.

(40) Andriamanampisoa, C.-L. et al. BIABooster: On-Line DNA Concentration and Size Profiling with a Limit of Detection of $10 \mathrm{fg} / \mu \mathrm{L}$. Application to High-Sensitivity Characterization of Circulating Cell-Free DNA. Anal. Chem. 2018, 90, 3766-3774.

(41) Viovy, J.-L. Electrophoresis of DNA and other polyelectrolytes: Physical mechanisms. Rev. Mod. Phys. 2000, 72, 813. 


\section{For Table of Contents Use Only}

Contraction and tumbling dynamics of DNA in shear flows under confinement induced by transverse viscoelastic forces

Marius Socol, Hubert Ranchon, Bayan Chami, Antony Lesage, Jean-Marc Victor,

Manoel Manghi, Aurélien Bancaud

Hydrodynamics
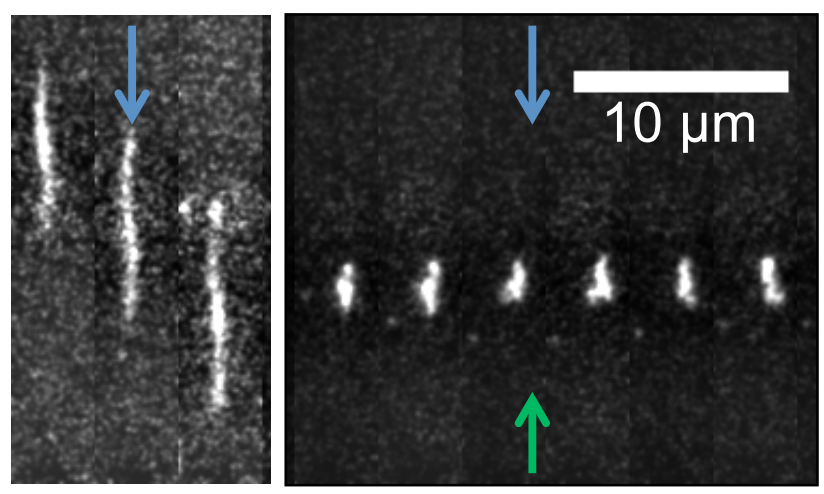

Electrophoresis 